\title{
On the phase of magneto-oscillations in graphite
}

\author{
L. Smrčka and N. A. Goncharuk \\ Institute of Physics, Academy of Science of the Czech Republic, v.v.i., \\ Cukrovarnická 10, 16253 Prague 6, Czech Republic
}

(Dated: December 4, 2018)

\begin{abstract}
The problem of Dirac fermions in graphite subject to a perpendicular magnetic field is studied. We show analytically that the weak inter-layer interaction between the graphene sheets leads to anomalies in the Shubnikov-de Haas and de Haas-van Alphen magneto-oscillations governed by the orbits around extremal cross-sections of the graphite Fermi surface. The calculation of the Landau plot performed within a four band continuum model reveals that magneto-oscillations are aperiodic, except of the case of vanishing inter-layer interaction at the $\mathrm{H}$ point of the graphite Brillouin zone. Also for all other orbits along the H-K-H edge the magneto-oscillations are only asymptotically periodic in the quasi-classical limit, with the phase corresponding to massive fermions.

PACS numbers: 71.20.-b, 71.70.Di, 81.05.Uw
\end{abstract}

Graphite is a layered material composed of weakly coupled two-dimensional (2D) graphene sheets formed by hexagonally arranged carbon atoms. In 2004, a single sheet of graphene was prepared from three-dimensional (3D) graphite by micro-mechanical cleavage [1]. The discovery immediately attracted attention of the solid-state physical community, as the electrons in graphene obey a linear energy dependence on the wave-vector $k$, and behave like massless relativistic particles - Dirac fermions (DFs). In the seminal papers [1, 2, 3], the Shubnikovde Haas $(\mathrm{SdH})$ magneto-oscillations in graphene were found periodic in an inverse magnetic field, $1 / B$, similarly as in the case of a 2D gas of massive Schrödinger fermions (SFs), but with the phase shifted by $\pi$. The shift, which was clearly demonstrated by the Landau plot of magneto-resistance oscillations, is due to the existence of the zero-energy Landau level (LL), shared by electrons and holes. For the same reason, the anomalous quantum Hall effect with a half-integer instead of integer quantization was observed in mechanically ex-foliated samples [2]. This is considered as the most direct evidence of DFs in graphene. In 2006, important technological progress was achieved. The epitaxial graphene was grown on the single-crystal silicon carbide by vacuum graphitization [4, 5].

The discovery of DFs in graphene has resulted in renewed interest in bulk graphite. In a series of papers [6, 7, 8], the spectral analysis of $\mathrm{SdH}$ and de Haas-van Alphen ( $\mathrm{dHvA}$ ) oscillations was employed to determine the phases of two series of magneto-oscillations observed in graphite. In papers [9, 10] an attempt was made to relate the phases to the topological Berry phase, which is acquired by fermions moving around close orbits. Based on their analysis the authors of Refs. [6, 7, 8] came to a conclusion that one of two groups of oscillating carriers corresponds to DFs. Recently a paper [11] was devoted to a careful, mostly experimental investigation of SdH effect in graphite, and doubts about the observation of DFs using magneto-transport measurements were expressed.

Here the problem is treated from the theoretical point of view. We construct a Landau plot for the model
Hamiltonian developed by Slonczewski, Weiss and MacClure (SWM) [12, 13, 14] and compare the result with Landau plots for SFs and DFs, described below.

The energy spectra of 2D SFs and DFs in a zero magnetic field can be written as

$$
E^{S}(k)=\frac{\hbar^{2} k^{2}}{2 m^{*}}, \quad E^{D}(k)= \pm \hbar v_{F} k
$$

where $k=\sqrt{k_{x}^{2}+k_{y}^{2}}, m^{*}$ is the effective mass of SFs and $v_{F}$ is the Fermi velocity of DFs. The positive and negative branches of the conical DFs spectrum correspond to the electrons and holes, respectively.

In a magnetic field, the spectra of SFs and DFs are quantized into the LLs as follows:

$$
E_{n}^{S}=\hbar \omega_{c}\left(n+\frac{1}{2}\right), \quad E_{n}^{D}= \pm \sqrt{2 \hbar|e| v_{F}^{2} B n}
$$

where $\omega_{c}=|e| B / m^{*}$ is the cyclotron frequency and the index $n=0,1,2, \cdots$. In the case of SFs the equidistant LLs lie above $E=0$ for any finite $B$, whereas in the DFs case the lowest electron LL is shared with the highest hole LL located exactly at $E=0$.

The Eqs. (2) are consistent with the Onsager-Lifshitz quasi-classical quantization rule

$$
A^{Q}\left(E_{F}\right)=\frac{2 \pi|e| B}{\hbar}\left(n+\gamma^{Q}\right), \quad Q=S, D,
$$

where $A^{Q}\left(E_{F}\right)=\pi k_{F}^{2}$ is the area of the SF or DF Fermi circle, calculated with the Fermi energy $E_{F}$ and the Fermi wave-vector $k_{F}$ taken from Eqs. (1). We get $\gamma^{S}=1 / 2$ for SFs and $\gamma^{D}=0$ for DFs.

Magneto-oscillations observed in $\mathrm{SdH}$ and dHvA effects are controlled by oscillations of the density of states (DOS). It is well known that the DOS on the Fermi level, $g\left(E_{F}\right)$, can be expressed as an imaginary part of the resolvent $G(z)=(z-H)^{-1}$,

$$
g\left(E_{F}\right)=-\frac{1}{\pi} \mathcal{I} m \operatorname{Tr} G\left(E_{F}+i 0\right) .
$$


For simple diagonal Hamiltonians of SFs and DFs given by Eqs. (2), we get

$$
g^{Q}\left(E_{F}\right)=\frac{|e| B}{2 \pi \hbar} \sum_{n=0}^{\infty} \delta\left(E_{F}-E_{n}^{Q}\right) .
$$

It follows from Eq. (5) that the DOS reaches maxima at magnetic fields $B_{n}$ for which the LLs cross the Fermi energy $E_{F}$. A Landau plot, i.e., the plot of the inverse magnetic fields $1 / B_{n}$ versus the level index $n$ is a standard tool used to determine the frequency and phase of magneto-oscillations. For SFs and DFs we arrive to

$$
\frac{B_{0}^{Q}}{B_{n}}=n+\gamma^{Q}
$$

where $B_{0}^{S}=m^{*} E_{F} /(\hbar|e|)$ and $B_{0}^{D}=E_{F}^{2} /\left(2 \hbar|e| v_{F}^{2}\right)$ are the oscillation frequencies, in agreement with the quasiclassical expression obtained from Eq. (3),

$$
B_{0}^{Q}=\hbar A^{Q} / 2 \pi|e| .
$$

It is clear that the positions of maxima of SF and DF oscillations differ by a half of the period, i.e., by $\pi$ in terms of a phase factor.

In graphite, the inter-layer interaction of Bernaldstacked graphenes adds a $k_{z}$-dependence to the electron energy spectrum and a 3D Fermi surface (FS) is formed close to the H-K-H edge of the hexagonal Brillouin zone (BZ). As mentioned above, the graphite 3D electronic structure is described by the semi-empirical SWM Hamiltonian, which employs seven nearest-neighbor tightbinding (TB) parameters $\gamma_{0}, \gamma_{1}, \ldots \gamma_{5}, \Delta$, and the value of the Fermi energy, $E_{F}$. Previously, the model parameters were fitted to various optical and transport experiments [15]. Recently, their values are continuously refined by fitting to the experimental data [11] and/or to the results of first-principles numerical simulations of the graphite band structure [16].

Among the seven SWM parameters, the parameter $\gamma_{3}$, which controls the trigonal warping of the FS, brings a numerical complications in the case of nonzero magnetic field. When $\gamma_{3}$ is taken into account, the magnetic-fieldSWM Hamiltonian has an infinite order and must be diagonalized numerically [17]. Fortunately, its influence is not too strong for $k_{z}$ far from the $\mathrm{H}$ point of the $\mathrm{BZ}$ and energies close to $E_{F}$ [17]. To facilitate our analytical treatment, we prefer to use a simplified Hamiltonian $H$ with $\gamma_{3}$ neglected, in the form introduced in the MacClure's paper [14].

The choice of $\gamma_{3}=0$ yields isotropic equienergetic contours. The FS of graphite consists of elongated electron and hole pockets located near the points $\mathrm{K}$ and $\mathrm{H}$, with $k_{z}$-dependent circular cross-sections. In the quasi-classical limit, two extremal cross-sections define two series of magneto-oscillations and two quasi-classical frequencies $B_{0}^{e}$ and $B_{0}^{h}$ for electrons and holes, respectively. Analytical solutions for the FS cross-sections can be found, e.g., in Ref. [15].

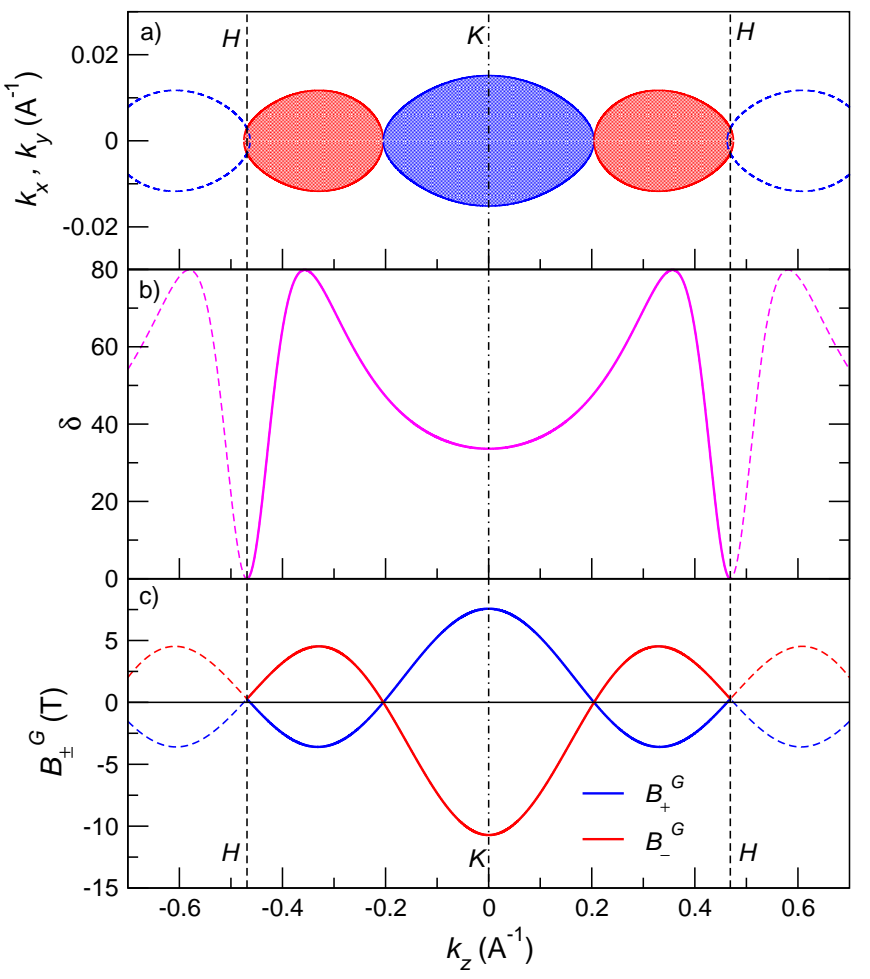

FIG. 1: (color online) a) Contours of the graphite Fermi surface, b) a dimensionless parameter $\delta$ as a function of $k_{z}, \mathrm{c}$ ) the parameters $B_{ \pm}^{G}$ as functions of $k_{z}$.

Within these approximations an expression similar to Eq. (6) can be obtained for any $k_{z}$-dependent crosssection of the 3D graphite FS. To do so, we need to find the poles of the resolvent $G(z)=\left(E_{F}-H+i 0\right)^{-1}$. In other words, we should solve the secular equation derived from the simplified Hamiltonian $H$ of Ref. [14] for $B_{n}$, i.e., we should find the roots $B_{n}$ of the secular polynomial.

The solution yields a formula for the inverse magnetic fields as a function of the level index, $n$, in a shape

$$
\frac{B_{ \pm}^{G}\left(k_{z}\right)}{B_{n}}=\frac{n+\frac{1}{2} \pm \sqrt{\frac{1}{4}+n(n+1) \delta\left(k_{z}\right)}}{1 \pm \sqrt{\delta\left(k_{z}\right)}},
$$

where the three coefficients $B_{ \pm}^{G}\left(k_{z}\right)$ and $\delta\left(k_{z}\right)$ can be derived from the SWM model and the value of $E_{F}$. The $k_{z}$-dependence originates from $\cos \left(k_{z} c / 2\right)$ which appears in the inter-layer TB SWM parameters, $c / 2$ denotes the inter-layer distance in graphite. Obviously, $B_{ \pm}^{G}\left(k_{z}\right) / B_{n}$, as given by Eq. (8), are not periodic in $1 / B$.

An expression for the Landau plot corresponding to Eq. (8) can be written in a form

$$
\frac{B_{ \pm}^{G}\left(k_{z}\right)}{B_{n}}=n+\gamma_{ \pm, n}^{G}\left(k_{z}\right) .
$$

In this equation $\gamma_{ \pm, n}^{G}\left(k_{z}\right)$, defined by the right-hand side of Eq. (8), is no longer a constant describing the oscilla- 


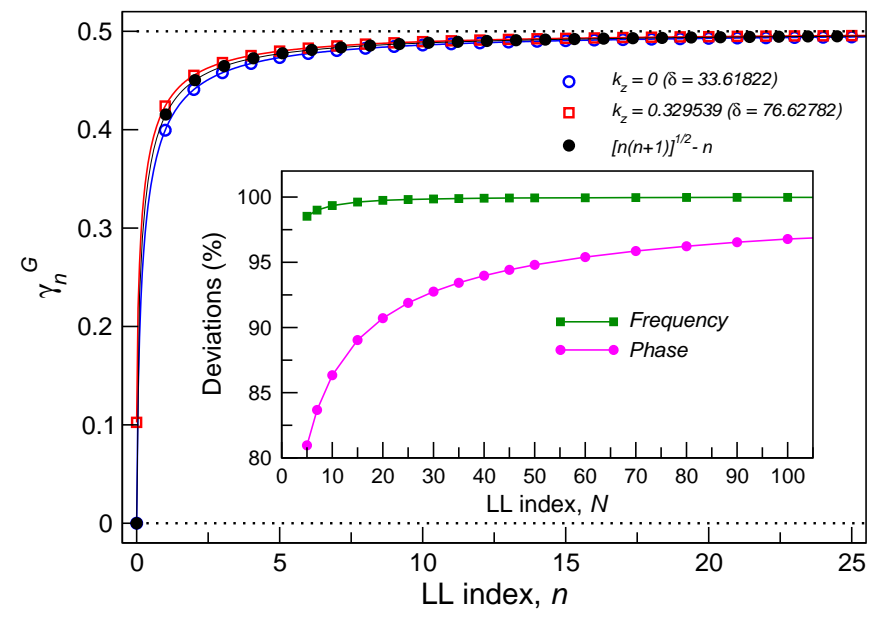

FIG. 2: (color online) $\gamma_{ \pm, n}^{G}\left(k_{z}\right)$ for maximum cross-sections of the electron and hole pockets approximated by $\sqrt{n(n+1)}-n$. The inset shows results of the linear approximation of first $N$ terms $(n=1,2, \cdots, N)$ of $\sqrt{n(n+1)}$. The relative deviations from the quasi-classical frequency and phase are shown.

tion phase, but a variable which depends on the LL index $n$.

The dependence on $n$ is most pronounced for high magnetic fields, i.e., for small $n$. In the low-magnetic-field limit, with a large number of LLs below $E_{F}$, we can write $n(n+1) \delta\left(k_{z}\right) \gg 1 / 4$ and $\sqrt{n(n+1)} \rightarrow n+1 / 2$. Then $\gamma_{ \pm, n}^{G}\left(k_{z}\right) \rightarrow 1 / 2$, and we can conclude that the charge carriers in graphite behave, at least as far as the phase is concerned, as the SFs.

Only when we can completely neglect the inter-layer interaction, as at the $H$ point of the $3 \mathrm{D} \mathrm{BZ,} k_{z}=\pi / c$, $\cos \left(k_{z} c / 2\right)=0, \Delta=0$ and $\delta\left(k_{z}\right) \rightarrow 0$, we get

$$
\frac{B_{ \pm}^{G}(\pi / c)}{B_{n}}=n+\frac{1}{2} \pm \frac{1}{2}
$$

a result which corresponds to DFs.

Here the Landau plots of two series of magnetooscillations, which can be observed in graphite, are considered as most interesting. We constructed them based on the parameters of the SWM model taken from Ref. [15]. The explicit expressions for $B_{ \pm}^{G}$ and $\delta\left(k_{z}\right)$, which appear in Eq. (8), will not be presented here. Instead, their $k_{z}$-dependences are shown in Fig. 1. together with the contours of the FS. The positive sign applies in the formula (8) for $k_{z}$ from the electron region of FS and the parameter $B_{+}^{G}$ is equal to the quasi-classical frequency $B_{0}^{e}$. Similarly, the negative sign should be taken for $k_{z}$ from the hole pockets, where $B_{-}^{G}$ becomes equal to $B_{0}^{h}$. For both electron and hole extremal orbits the parameter $\delta$ is large and, consequently, the right-hand side of Eq. (8) can be approximated by $\sqrt{n(n+1)}$. The marked difference is only for $n=0$. The Fig. 2 shows to what extent the accuracy of this approximation is reasonable.

The expression for $B_{ \pm}^{G} / B_{n}$ is not periodic and the question arises how many LLs must be resolved to reach the linear dependence on $n$, i.e., the quasi-classical limit $n+1 / 2$. The result of the linear approximation of a model curve $\sqrt{n(n+1)}$ is presented in the inset of Fig. 2, where the relative deviations from the quasi-classical frequency and phase are shown. It turns out that both frequency and phase are underestimated if we took into account only limited number of $n, 1 \leq n \leq N$. Less oscillations are necessary to get close to the quasi-classical frequency than to obtain a reasonable approximation for the phase. This may explain the differences found between the experimentally determined phases of samples with different mobilities, which are determined from different number of oscillations resolved.

Note that the above Landau plot can be derived if we approximate the energy spectra of electrons and holes by the formulae

$$
E_{n}^{e}=\hbar \omega_{c}^{e} \sqrt{n(n+1)}, \quad E_{n}^{h}=\hbar \omega_{c}^{h} \sqrt{n(n+1)},
$$

where $\omega_{c}^{e}$ and $\omega_{c}^{h}$ are the quasi-classical cyclotron frequencies corresponding to extremal electron and hole orbits.

Two series of magneto-oscillations discussed above correspond to $\delta \gg 1$ and to the maximum cross-sections of the electron and hole pockets. There are another two extremal cross-sections, till now not reliably resolved in the transport experiments, located around the $\mathrm{H}$ point of the $\mathrm{BZ}$ where $\delta \leq 1$. In spite of the fact that, according to Ref. [17], the parameter $\gamma_{3}$ has a qualitative influence on the LLs structure near this point, it is at least of the theoretical interest to study the behavior of the $\gamma_{ \pm, n}^{G}\left(k_{z}\right)$ for model with $\gamma_{3}$ neglected.

For a given $k_{z}$ the dependence of the energy bands on $k$ is hyperbolic in a zero magnetic field. While near the maximum cross-sections there are broad minima/maxima of bands which are similar to parabolas for small $k$, near the $\mathrm{H}$ point the hyperbolas are very sharp and with a shape close to the Dirac cone. Therefore,

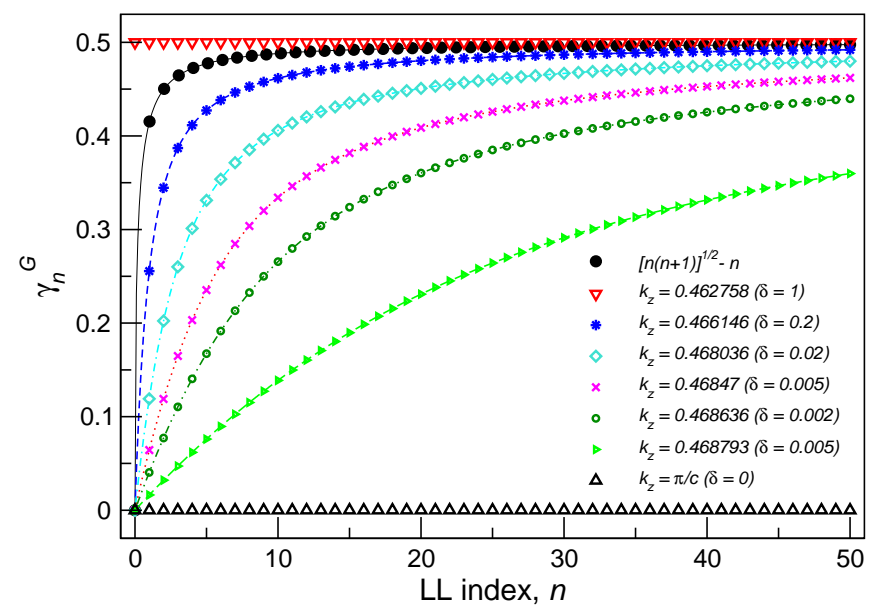

FIG. 3: (color online)The dependence of $\gamma_{-, n}\left(k_{z}\right)$ on the LL index $n$ for $k_{z}$ close to the $\mathrm{H}$ point of the BZ. 
a smooth transition of $\gamma_{ \pm, n}^{G}\left(k_{z}\right)$ from SFs to DFs is expected. Fig. 3 reveals that the behavior is more complicated.

In the SWM model the $k$-dependence of the zero-field energy at the $\mathrm{H}$ point $\left(k_{z}=0\right)$ is given by

$$
E^{G}=\frac{\Delta}{2} \pm \sqrt{\left(\frac{\Delta}{2}\right)^{2}+\hbar^{2} v_{F}^{2} k^{2}},
$$

which is not equal to the Dirac cone for a finite $\Delta$. Nevertheless, $\delta=0$ implies that $\gamma_{-, n}^{G}\left(k_{z}\right)$ is a constant equal to 0 , as for the DFs. This is in agreement with the Landau plot constructed from the energy spectra in a magnetic field, which according to Ref. [14] have a simple analytic form

$$
E_{n}^{G}=\frac{\Delta}{2} \pm \sqrt{\left(\frac{\Delta}{2}\right)^{2}+2 \hbar|e| v_{F}^{2} B n}
$$

On the other hand, the parameter $\delta$ equals 1 for $E_{F}$ crossing the $E_{2}$ band of the SWM model. According to Eq. (8) this leads to $\gamma_{-, n}^{G}\left(k_{z}\right)=1 / 2$, as for the SFs, in spite of the $k$-dependence not so close to parabolic one as the for the maximum cross-sections, $\delta \gg 1$, where the energy spectra can be approximated by Eqs. (11).

The field dependence of the corresponding LLs ranges from that described by Eq. (13) at the $\mathrm{H}$ point, which is close to $\sqrt{B}$ characteristic for DFs, to the linear dependence on $B$ typical for SFs for extremal electron and hole orbits, as given by Eqs. (11).

It follows from the above discussion that the hyperbolic $k$-dependence of the zero-field electron energy bands, which changes considerably depending on the value of $k_{z}$, yields aperiodic magneto-oscillations when a magnetic field is applied. An exception are two $k_{z}$ in the neighborhood of the $\mathrm{H}$ point of the BZ. We assume that this conclusion is at least qualitatively correct, as one can hardly believe that this is just the neglected $\gamma_{3}$ which yields the magneto-oscillation aperiodicity.

There is another potential reason for deviations from the magneto-oscillation periodicity. Unlike the optical experiments which involve electrons with energies below and above the Fermi energy, the $\mathrm{SdH}$ and dHvA magnetooscillations reflect only the properties of electrons with an energy equal to $E_{F}$. Our treatment is based on the assumption that the $E_{F}$ is a constant. This is not quite correct as the carrier concentration is a constant and not $E_{F}$, which should oscillate as a function of $B$. This can be important for lowest LLs in high mobility samples and was considered as a single source of oscillation aperiodicity in Ref. [11].

In conclusion, we have found that the magnetooscillations in graphite are only asymptotically periodic in the quasi-classical limit, with the phase corresponding to massive fermions. The quasi-classical limit can be reached only exceptionally for samples with very high mobility and at very low magnetic field. Therefore, the determination of the oscillation phase in samples with a limited number of resolved LLs below $E_{F}$ is not a reliable tool for distinguishing between DFs and SFs in graphite, due to the aperiodicity of the magneto-oscillations in a standard quantum regime.

The authors acknowledge the support of the Academy of Sciences of the Czech Republic project KAN400100652 and the Ministry of Education of the Czech Republic project LC510.
[1] K. S. Novoselov, A. K. Geim, S. V. Morozov, Y. Z. D. Jiang, S. V. Dubonos, I. V. Grigorieva, and A. A. Firsov, Science 306, 666 (2004).

[2] K. S. Novoselov, A. K. Geim, S. V. Morozov, D. Jiang, M. I. Katsnelson, I. V. Grigorieva, S. V. Dubonos, and A. A. Firsov, Nature 438, 197 (2005).

[3] Y. Zhang, Y.-W. Tan, H. L. Stormer, and P. Kim, Nature 438, 201 (2005).

[4] C. Berger, Z. Song, X. Li, X. Wu, N. Brown, C. Naud, D. Mayou, T. Li, J. Haas, A. N. Marchenkov, et al., Science 312, 1191 (2006).

[5] C. Berger, Z. Song, X. Li, X. Wu, N. Brown, D. K. Maude, C. Naud, and W. A. de Heer, phys. stat. sol. (a) 204, 1746 (2007).

[6] I. A. Luky'anchuk and Y. Kopelevich, Phys. Rev. Lett. 93, 166402 (2004).

[7] I. A. Luky'anchuk and Y. Kopelevich, Phys. Rev. Lett. 97, 256801 (2006).

[8] I. A. Luky'anchuk, Y. Kopelevich, and M. E. Marssi,
Physica B 404, 404 (2009).

[9] G. P. Mikitik and Y. V. Sharlai, Phys. Rev. Lett. 82, 2147 (1999).

[10] G. P. Mikitik and Y. V. Sharlai, Phys. Rev. B 73, 235112 (2006).

[11] J. M. Schneider, M. Orlita, M. Potemski, and D. K. Maude, arXiv:0902.1925.

[12] C. Slonczewski and P. R. Weiss, Phys. Rev. 109, 272 (1958).

[13] J. W. McClure, Phys. Rev. 108, 612 (1957).

[14] J. W. McClure, Phys. Rev. 119, 606 (1960).

[15] M. S. Dresselhaus and G. Dresselhaus, Advances in Phys. 30 30, 139 (1981).

[16] A. Gruneis, C. Attaccalite, L. Wirtz, H. Shiozawa, R. Saito, T. Pichler, and A. Rubio, Phys. Rev. B 78, 205425 (2008).

[17] K. Nakao, J. Phys. Soc. Japan 40, 761 (1976). 